Review

\title{
The role of YWHAZ in cancer: A maze of opportunities and challenges
}

\author{
Yun Gan ${ }^{1}$, Feng $\mathrm{Ye}^{2 \bowtie}$, Xing-Xing $\mathrm{He}^{1 凶}$ \\ 1. Institute of Liver and Gastrointestinal Diseases, Tongji Hospital, Tongji Medical College, Huazhong University of Science and Technology, Wuhan, China \\ 2. Department of Pediatrics, Tongji Hospital, Tongji Medical College, Huazhong University of Science and Technology, Wuhan, China \\ $\triangle$ Corresponding author: Dr. Xing-Xing He, Institute of Liver and Gastrointestinal Diseases, Tongji Hospital, Tongji Medical College, Huazhong University of \\ Science and Technology, Wuhan 430030, China. Tel/Fax: 86-27-69378506, E-mail: xxhe@tjh.tjmu.edu.cn; or Dr. Feng Ye, Department of Pediatrics, Tongji \\ Hospital, Tongji Medical College, Huazhong University of Science and Technology, Wuhan 430030, China. Tel/Fax: 86-27-69378504, E-mail: 280682605@qq.com. \\ (C) The author(s). This is an open access article distributed under the terms of the Creative Commons Attribution License (https://creativecommons.org/licenses/by/4.0/). \\ See http://ivyspring.com/terms for full terms and conditions.
}

Received: 2019.10.19; Accepted: 2019.11.30; Published: 2020.02.03

\begin{abstract}
YWHAZ (also named 14-3-3ל) is a central hub protein for many signal transduction pathways and plays a significant role in tumor progression. Accumulating evidences have demonstrated that $Y W H A Z$ is frequently up-regulated in multiple types of cancers and acts as an oncogene in a wide range of cell activities including cell growth, cell cycle, apoptosis, migration, and invasion. Moreover, YWHAZ was reported to be regulated by microRNAs (miRNAs) or long non-coding RNAs and exerted its malignant functions by targeting downstream molecules like protein kinase, apoptosis proteins, and metastasis-related molecules. Additionally, YWHAZ may be a potential biomarker of diagnosis, prognosis and chemoresistance in several cancers. Targeting YWHAZ by siRNA, shRNA or miRNA was reported to have great help in suppressing malignant properties of cancer cells. In this review, we perform literature and bioinformatics analysis to reveal the oncogenic role and molecular mechanism of YWHAZ in cancer, and discuss the potential clinical applications of YWHAZ concerning diagnosis, prognosis, and therapy in malignant tumors.
\end{abstract}

Key words: YWHAZ, cancer, function, molecular mechanism, biomarker

\section{Introduction}

14-3-3 proteins, which have a molecular mass of around $30 \mathrm{kDa}$, are a family of highly conserved molecules [1]. Seven 14-3-3 isoforms are known to exist $-\beta, \gamma, \varepsilon, \eta, \sigma, \theta$, and $\zeta$ - each of which localizes distinctly in tissues with independent isoform-specific functions [1-4]. Tyrosine 3 monooxygenase/tryptophan 5-monooxygenase activation protein zeta (also named 14-3-3 3 or YWHAZ), belonging to the 14-3-3 protein family, is a central hub protein involved in many signal transduction pathways and plays a key role in tumor progression [1, 2, 5-8]. A growing body of research has demonstrated that YWHAZ was frequently up-regulated and participated in a wide range of cell activities including cell growth, cell cycle, apoptosis, migration/invasion in multiple types of cancers, such as hepatocellular carcinoma, colorectal cancer, lung cancer and breast cancer [5-8]. In this review, we seek to summarize the oncogenic role and molecular regulatory network of YWHAZ, with the aim of discovering potential clinical applications of YWHAZ regarding diagnosis, prognosis and treatment in malignant tumors.

\section{Expression and functions of YWHAZ in cancer}

Growing researches have reported that YWHAZ is frequently up-regulated in multiple types of cancers, acting as an oncogene by promoting malignant properties of cancer cells (summarized in Table 1). Using UALCAN database [9], we analyzed the expression of YWHAZ in tumor tissues and adjacent tissues from nine types of high-morbidity cancers and observed that YWHAZ was significantly increased in breast carcinoma (BRCA), colon adenocarcinoma (COAD), esophagus carcinoma (ESCA), liver hepatocellular carcinoma (LIHC), lung 
adenocarcinoma (LUAD), lung squamous carcinoma (LUSC) and stomach adenocarcinoma (STAD) $(\mathrm{p}<$ 0.0001). However, in prostate adenocarcinoma (PRAD) and rectum adenocarcinoma (READ), there was no significant difference in the expression of YWHAZ between cancer tissues and adjacent tissues (Figure 1).

Table 1. Functions and relevant molecular mechanisms of YWHAZ

\begin{tabular}{|c|c|c|c|c|c|}
\hline Cancer type & Function of YWHAZ & YWHAZ complex & $\begin{array}{l}\text { Upstream regulators } \\
\text { of YWHAZ }\end{array}$ & $\begin{array}{l}\text { Downstream } \\
\text { targets } \\
\text { of YWHAZ }\end{array}$ & Reference \\
\hline $\begin{array}{l}\text { Hepatocellular } \\
\text { carcinoma }\end{array}$ & $\begin{array}{l}\text { Enhanced cell proliferation, colony } \\
\text { formation, migration/invasion, EMT, } \\
\text { chemoresistance; Inhibited cell apoptosis }\end{array}$ & $\begin{array}{l}\text { Bound with } \\
\text { aB-Crystallin; Axl; HO-1 }\end{array}$ & $\begin{array}{l}\text { miR-22; miR-375; } \\
\text { miR-451a; miR-613 }\end{array}$ & $\begin{array}{l}\text { AKT; ERK1/2; Caspase-3; Bax; Smad3; } \\
\text { TGF- } \beta \text {; HDCA4; HIF-1a; JNK and P38; } \\
\text { STAT3; ATG7;P53;E-cadherin }\end{array}$ & $\begin{array}{l}{[5,10-16} \\
60,71,85]\end{array}$ \\
\hline $\begin{array}{l}\text { Colorectal } \\
\text { cancer }\end{array}$ & $\begin{array}{l}\text { Promoted cell growth, colony formation, } \\
\text { migration, invasion, EMT }\end{array}$ & Interacted with TRIP13 & $\begin{array}{l}\text { miR-451; LINC00858; } \\
\text { miR-22-3p }\end{array}$ & $\begin{array}{l}\text { FoxO3; N-cadherin; } \beta \text {-catenin; snail; } \\
\text { E-cadherin }\end{array}$ & $\begin{array}{l}{[6,18,19,} \\
63]\end{array}$ \\
\hline $\begin{array}{l}\text { Gastric } \\
\text { carcinoma }\end{array}$ & $\begin{array}{l}\text { Promoted cell proliferation, } \\
\text { migration/invasion, EMT; Inhibited cell } \\
\text { apoptosis }\end{array}$ & / & $\begin{array}{l}\text { miR-375; LUCAT1; } \\
\text { miR-134-5p }\end{array}$ & $\begin{array}{l}\text { PDK1/Akt; Caspase-3; Caspase-7; } \\
\text { wnt/ } \beta \text {-catenin; E-cadherin; N-cadherin; } \\
\text { Vimentin; PI3K/AKT/mTOR }\end{array}$ & {$[20-23,61]$} \\
\hline Lung cancer & $\begin{array}{l}\text { Promoted cell proliferation, EMT, } \\
\text { migration/invasion; Inhibited cell apoptosis }\end{array}$ & $\begin{array}{l}\text { Bound with heat shock } \\
\text { protein27; } \beta \text {-catenin; } \\
\text { Par3; Tiam1 }\end{array}$ & / & $\begin{array}{l}\beta \text {-catenin; Protein kinase C/NF-kB and } \\
\text { Snail; E-cadherin; N-cadherin; Vimentin; } \\
\text { TGF } \beta \text { R1; MUC1 }\end{array}$ & {$[7,24-30]$} \\
\hline Breast cancer & $\begin{array}{l}\text { Induced cell proliferation, colony formation, } \\
\text { metastasis / invasion, chemoresistance; } \\
\text { Inhibited cell apoptosis }\end{array}$ & Bound with ErbB2; p85 & $\begin{array}{l}\text { miR-193b; miR-451; } \\
\text { miR-30c }\end{array}$ & $\begin{array}{l}\text { Caspase-3;Bax; PI3K/Akt; TGF-b/ Smad; } \\
\text { ZFHX1B; TbRI; FOXM1; HER2; EGFR; } \\
\text { MAPK; miR-221; c-Jun; } \beta \text {-catenin }\end{array}$ & $\begin{array}{l}{[8,31,32,} \\
34-40,54 \\
58]\end{array}$ \\
\hline Prostate cancer & $\begin{array}{l}\text { Promoted cell proliferation, colony } \\
\text { formation, migration/invasion; Inhibited cell } \\
\text { apoptosis }\end{array}$ & / & / & Rac1 & {$[41-44]$} \\
\hline
\end{tabular}

Expression of YWHAZ in BRCA based on sample types

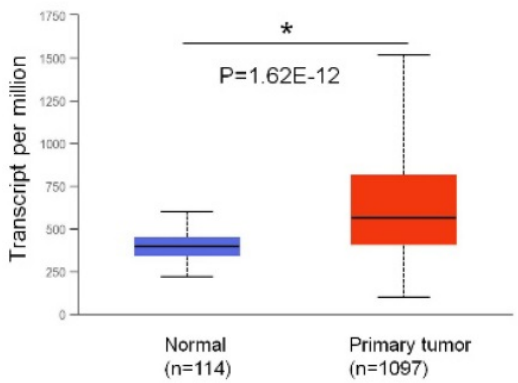

Expression of YWHAZ in $\mathrm{LIHC}$ based on sample types

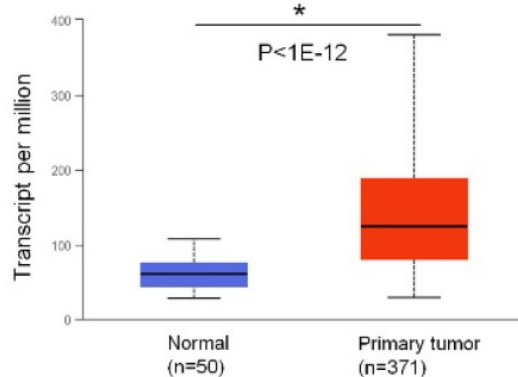

Expression of YWHAZ in PRAD based on sample types

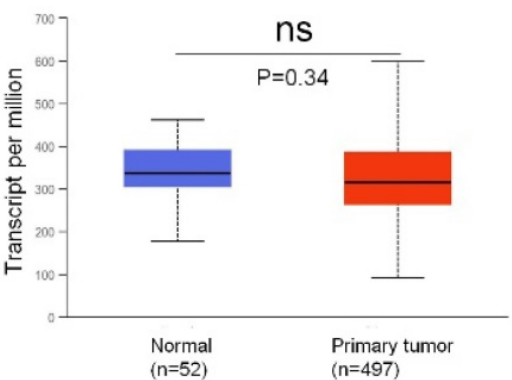

Expression of YWHAZ in COAD based on sample types

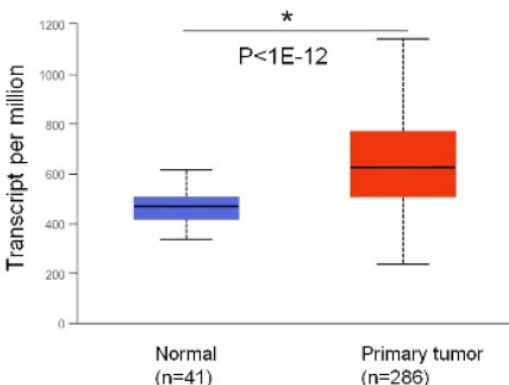

Expression of YWHAZ in LUAD based on sample types

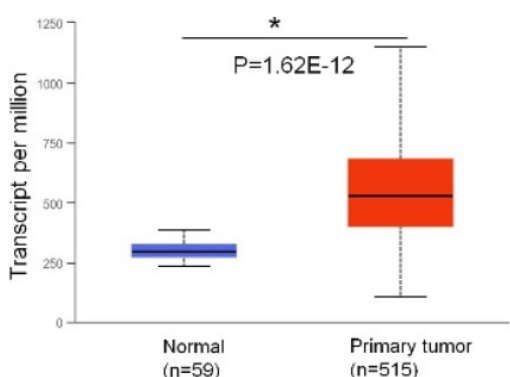

Expression of YWHAZ in READ based on sample types

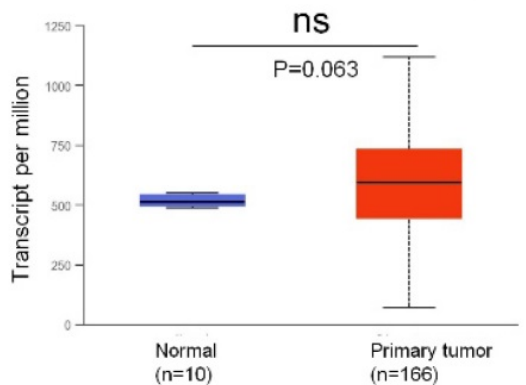

Expression of YWHAZ in ESCA based on sample types

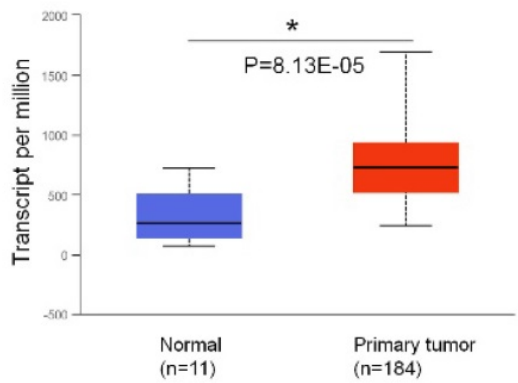

Expression of YWHAZ in LUSC based on sample types

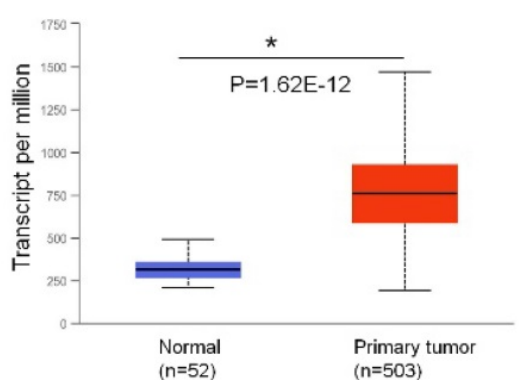

Expression of YWHAZ in STAD based on sample types

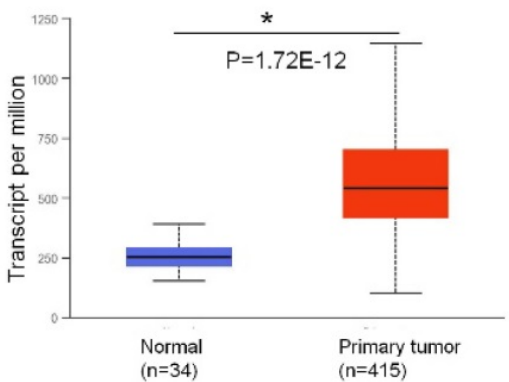

Figure 1. The mRNA level of YWHAZ in multiple types of cancers in TCGA samples from UALCAN database. * represents significant difference between two groups. BRCA: breast carcinoma; COAD: colon adenocarcinoma; ESCA: esophagus carcinoma; LIHC: liver hepatocellular carcinoma; LUAD: lung adenocarcinoma; LUSC: lung squamous carcinoma; PRAD: prostate adenocarcinoma; READ: rectum adenocarcinoma; STAD: stomach adenocarcinoma. 


\section{Hepatocellular carcinoma}

We previously examined the mRNA level of YWHAZ in 53 pairs of hepatocellular carcinoma (HCC) tissues and adjacent tissues and found that YWHAZ was significantly up-regulated in HCC tissues [5]. Similarly, YWHAZ mRNA expression was higher in eight liver cancer cell lines than normal liver cell line [5]. Zhao JF et al. and Chen $\mathrm{M}$ et al. likewise verified the high mRNA level of YWHAZ in $50 \mathrm{HCC}$ tissues and $374 \mathrm{HCC}$ tissues respectively from The Cancer Genome Atlas (TCGA) database [5, 10]. Additionally, YWHAZ protein expression was also higher in 11 of 12 HCC tissues and 8 liver cancer cell lines by western blot and was enhanced in 72 of 135 HCC tissues by immunohistochemical (IHC) [5, 11]. Besides, YWHAZ protein level was higher in 10 portal vein tumor thrombus (PVTT) $(+)$ tumors than that in PVTT (-) tumors [12].

Previously, we performed gain- and loss-of-function experiments in liver cancer cells, demonstrating that YWHAZ silencing decreased cell proliferation, clonogenicity, migration/invasion and induced G2 arrest and apoptosis, while YWHAZ up-regulation led to the opposite [5]. Choi JE et al. showed that YWHAZ knockdown increased the chemotherapeutic effect of cis-diammined dichloridoplatium through phosphorylation of JNK and p38 [11]. Lee YK et al. revealed that YWHAZ silencing in liver cancer stem-like cells reduced radio-resistance, leading to decreased cell viability and enhanced apoptosis following $\gamma$-irradiation [13]. Under both normoxic and hypoxic conditions, down-regulation of YWHAZ reduced invasion capacity, which could be rescued by hypoxia-induced factor-1a (HIF-1a) [12]. Furthermore, YWHAZ could exert malignant functions by forming complexes with other molecules in HCC [14-16]. aB-Crystallin (Cryab) protein, an oncoprotein belonging to the mammalian small heat shock protein family and related with cellular physiology and growth, was up-regulated and formed a complex with YWHAZ, inducing epithelial-mesenchymal transition (EMT) via ERK1/2/Fra-1/slug signaling [14]. In Addition, YWHAZ could bind to Axl, promoting Axl-mediated cell migration and invasion [15]. However, YWHAZ interference dismissed the mesenchymal phenotype conferred by Cryab overexpression and decreased Gas6/Axl-dependent migration and invasion [14, 15]. Song $J$ et al. reported that YWHAZ interacted with heme oxygenase 1 (HO-1) and stabilized HO-1 protein expression by inhibiting its ubiquitin-mediated degradation [16]. YWHAZ/HO-1 complex promoted HCC proliferation by signal transducers and activators of transcription 3 (STAT3) signaling pathway [16]. Based on the above studies, it may be inferred that YWHAZ overexpression was implicated in HCC progression.

\section{Colorectal cancer}

$\mathrm{Li} Y$ et al. observed that the mRNA and protein levels of YWHAZ were both increased in 46 colorectal cancer (CRC) tissues by qRT-PCR and IHC [6]. Likewise, YWHAZ protein expression was 1.3-fold higher in COAD stromal tissues than non-cancer stromal tissues by isobaric tags for relative and absolute quantitation-based quantitation proteomics [17]. MiR-451 was down-regulated in colon cancer, and its expression was inversely correlated with YWHAZ, which promoted cell growth through suppression of the nuclear accumulation of FoxO3 [6]. Additionally, YWHAZ may be responsible for conferring malignant phenotype via extracellular vesicles, while YWHAZ silencing significantly decreased colony formation in CRC cells [18]. Thyroid hormone receptor interactor 13 (TRIP13) was reported to interact with YWHAZ and mediate EMT in CRC [19]. Knockdown of YWHAZ in TRIP13-overexpressing CRC cells inhibited migration and invasion abilities, as well as decreasing the expression of $\mathrm{N}$-cadherin, $\beta$-catenin, snail and increasing the expression of E-cadherin [19].

\section{Gastric cancer}

Guo $\mathrm{F}$ et al. reported that the mRNA and protein level of YWHAZ were higher in four gastric cancer (GC) cell lines [20]. Enhanced YWHAZ expression was also detected in 6 of 7 GC cell lines $(85.7 \%)$ by western blot and in 72 of 141 primary GC samples (51\%) by IHC [21]. In GC, YWHAZ was downregulated in cells transfected with miR-375 and luciferase reporter indicated that miR-375 targets the 3' UTR of YWHAZ [20, 22]. Silencing of YWHAZ accelerated $m i R$-375-induced apoptosis by caspase-3/ caspase-7 activation and promoted autophagy by $\mathrm{PI} 3 \mathrm{~K} / \mathrm{AKT} / \mathrm{mTOR}$ signaling pathway $[22,23]$, as well as inhibiting cell proliferation, migration/invasion and EMT in GC [20,21].

\section{Lung cancer}

Deng $\mathrm{Y}$ et al. reported that YWHAZ mRNA and protein expression was significantly higher in 152 non-small cell lung cancer (NSCLC) tissues compared to 30 noncancerous lung tissues by qRT-PCR and IHC [24]. Using western blot, Zhao G-Y et al. also detected higher YWHAZ expression in 16 NSCLC tissues than in matched adjacent tissues [25]. Chen $\mathrm{CH}$ et al. observed that YWHAZ copy number, mRNA and protein expression were all higher in highly invasive lung cancer cell line than less invasive lung cancer cell line [26]. Besides, YWHAZ mRNA and protein expression were higher in positive lymph node LUSC 
patients than that in negative lymph node patients [27].

In vitro, proliferation, migration/invasion and EMT were enhanced in lung cancer cells overexpressing YWHAZ [7, 26, 28], while silence of YWHAZ led to the opposite [24, 27, 29]. Immunoprecipitation and immunofluorescence analysis revealed that YWHAZ formed complex with Hsp27 protein, colocalizing in the cytoplasm of lung cancer cells [25]. Knockdown of this complex suppressed migration of lung cancer cells [25]. Additionally, YWHAZ bound with partitioning defective protein 3 (Par3) in lung cancer and loss of Par3 enhanced the interaction of YWHAZ and Tiam1, subsequently activating Rac1 and promoting cancer cell metastasis [30]. To confirm YWHAZ function in vivo, Chen $\mathrm{CH}$ et al. performed three approaches: 1) YWHAZ-cell and control-cell were subcutaneously implanted into the dorsal regions of severe combined immunodeficiency (SCID) mice; 2) YWHAZ-cell and control-cell were injected directly into the circulation of SCID mice to bypass the initial steps of local invasion and intravasation; 3) YWHAZ-cell and control-cell were orthotopically injected into one lobe of SCID mouse lung [26]. Tumorigenesis at injection site, local metastasis to the adjunct lobe of the lung, and distant metastasis to the liver were all significantly increased in mice undergone injection of YWHAZ-expressing clone cells [26]. Results from these approaches support a role for YWHAZ in promoting cancer metastasis [26]. Based on the above studies, malignant transformation of cells induced by increased YWHAZ has been strikingly elucidated in lung cancer.

\section{Breast cancer}

YWHAZ protein expression was assessed by IHC in 139 BRCA tissues and was found to be higher in $45 \%$ of BRCA specimens [31]. Likewise, Neal CL et al. reported that YWHAZ IHC staining was strongly positive in $42 \%(\mathrm{n}=51 / 121)$ of invasive BRCA specimens [32]. TCGA RNA-seq data of 104 corresponding BRCA samples revealed up-regulated YWHAZ in cancer tissues compared with adjacent normal tissues [8]. Additionally, YWHAZ expression was substantially increased in tamoxifen-resistant BRCA cells compared with chemo-sensitive cells [33].

In BRCA, increased YWHAZ expression had been reported to induce anchorage-independent growth, malignant transformation of cancer cells, and resistance to apoptosis via inhibition of the mitochondrial apoptotic pathway [32]. However, Knockdown of YWHAZ greatly decreased cell growth, proliferation, invasion capacity, as well as enhancing tamoxifen-induced inhibition of cell viability and apoptosis promotion [31, 32, 34, 35].
Furthermore, YWHAZ can bind to serine 83 on p85, contributing to transformation-related properties of BRCA cells [36]. Inhibition of YWHAZ binding to p85 was found to reduce cell proliferation and promote apoptosis [36]. In in vivo studies, YWHAZ overexpression in FVB mice accelerated the progression of mammary tumors through EMT, angiogenesis promotion and apoptosis inhibition [37]. Conversely, delayed tumor onset and reduced tumor growth were observed in mice injected with YWHAZ siRNAtreated cells compared with siRNA-control cells [32]. To date, combinations of YWHAZ and several oncogenic molecules had been considered to promote transition to invasive breast cancer [38-40]. YWHAZ overexpression disrupted the architecture of mammary epithelial cell acini in 3-dimensional culture, resulting in luminal filling, which is a feature of early-stage, benign breast epithelial lesions [38]. This progression may be attributed to p53 proteasomal degradation-induced anoikis resistance via the YWHAZ-PI3K-Akt pathway [38]. Lu J et al. identified that 8 of the 25 cases $(32 \%)$ exhibited high levels of both ErbB2 and YWHAZ [39]. ErbB2-mediated increase in cell migration and YWHAZ-mediated decrease in cell-cell adhesion via EMT were found to enhance acini invasiveness [39]. Co-overexpression of both molecules was considered requisite to induce full transformation, but overexpression of one of these molecules alone was not sufficient to promote progression from ductal carcinoma in situ (CIS) to invasive BRCA and metastasis [39]. Unexpectedly, Kambach DM et al. demonstrated that ionizing radiation, oxidative stress and Src-mediated induction of YWHAZ were all capable of inducing invasion of FoxM1-positive cells, even in the absence of ErbB2 expression [40]. In summary, these findings strongly support the oncogenic nature of YWHAZ in the promotion of BRCA progression.

\section{Prostate cancer}

In prostate cancer, YWHAZ protein expression was observed to be significantly higher in tumorigenic/metastatic prostate cell lines compared with non-tumorigenic cell line and higher in 50 of 90 prostate cancer tissues than in benign prostate tissues [41, 42]. YWHAZ mRNA levels showed consistency with protein level [41]. It was suggested, through assessment of somatic copy number alterations and IHC, that YWHAZ was noticeably amplified and up-regulated in castration-resistant prostate cancer (CRPC) cases compared with non-CRPC patients [43, 44]. Murata T et al. reported that YWHAZ mRNA and protein level was both up-regulated by androgen stimulation [41]. Moreover, YWHAZ was associated with the androgen receptor (AR) in the nucleus, 
promoting AR transcriptional activity [41]. Overexpression of YWHAZ promoted cell proliferation and migration in prostate cancer cells, while silencing of YWHAZ showed the opposite [41,43]. It is well known that YWHAZ dimerization is tightly correlated with its activity in cells, proven to be upstream of rac1 activation [42, 45]. Dimerization of increased YWHAZ was found to significantly enhance cell proliferation, viability, and colony formation, while YWHAZ/rac1 complex promoted cell-matrix interactions, lamellipodia formation, cell migration in prostate cancer cell lines [42].

\section{Other tumors}

In acute myeloid leukemia (AML), YWHAZ protein expression was increased in 29 AML patients compared with 24 healthy donors [46]. Liang $\mathrm{R}$ et al. indicated that YWHAZ mRNA and protein expression was obviously higher in vincristine drug-resistant AML cell line than in AML-sensitive cell line [47]. Knockdown of YWHAZ by siRNA effectively reduced cell growth and proportion of cells in the S/G2 phases, while increasing the proportion of cells in the G0/G1 phase and enhancing sensitivity to topotecan in both drug-resistant and sensitive AML cells [47]. In intrahepatic cholangiocarcinoma (ICC), western blot showed that the protein level of YWHAZ was significantly higher in 30 ICC tissues, and IHC further confirmed the enhanced YWHAZ protein expression in 120 ICC samples [48]. Overexpression of YWHAZ was positively related with lymphatic metastasis, tumor-node-metastasis stage, recurrence and the expression of EMT-related markers in ICC [48]. Reversely, silence of YWHAZ impaired the invasion, migration, and proliferation of ICC cells [48]. In diffuse large B cell lymphoma (DLBCL), 20 of 35 DLBCL cases showed positive expression of YWHAZ and higher YWHAZ was also found in the metastatic T1 DLBCL lymph node tissue compared with the non-metastatic DLBCL tissue and a normal lymph node [49]. Moreover, chemotherapeutic mixture consisting of cyclophosphamide, doxorubicin, vincristine, and prednisone (CHOP)-resistant DLBCL cells expressed markedly higher levels of YWHAZ than CHOP-sensitive cells [49]. Further study demonstrated that blockade of YWHAZ inhibited DLBCL cell growth, leading to the accumulation of cells in the G2/M phase and restoring the sensitivity of resistant DLBCL to CHOP-induced apoptosis [49].

In summary, YWHAZ is frequently up-regulated in various cancers, functioning as an oncogene by promoting the malignant phenotype of cancer cells, particularly through acceleration of migration and invasion.

\section{Signaling pathways associated with YWHAZ in cancer}

\section{Upstream regulators of YWHAZ}

MiRNAs are small, non-coding RNAs of 20-22 nucleotides in length, which are considered to be vital components of gene regulation as important as transcription factors [50]. Alterations and dysregulation of miRNAs are often implicated in the initiation and progression of human cancers and are essential for maintaining the malignant phenotype of cancer cells [51, 52]. Our team previously proved that YWHAZ was a downstream target of miR-375 and YWHAZ expression was negatively correlated with $m i R-375$ in HCC [5]. Ectopic expression of miR-375 resulted in decreased YWHAZ, subsequently accelerating caspase-related apoptosis in gastric carcinoma and repressing telomerase activity in HPV-associated cancers [22, 53]. Furthermore, YWHAZ expression was enhanced by miR-451 down-regulation, subsequently regulating a series of cell activities, including cell proliferation, survival, apoptosis and endocrine chemoresistance $[6,8,33,46$, 54]. For example, low miR-451/high YWHAZ expression was observed to promote cell proliferation and inhibit apoptosis through AKT targeting in AML and activate growth factor receptors and kinases (HER2, EGFR, AKT, and MAPK) involved in endocrine resistance in breast cancer $[46,54]$. Conversely, negative regulation of $Y W H A Z$ via high $m i R-451$ expression greatly reduced cell proliferation and growth and induced cell-cycle arrest alongside apoptotic cascade in breast cancer $[8,54]$. Li Y et al. elucidated that decreased $Y W H A Z$ expression via miR-451 activity inhibited cell growth in colorectal cancer through nuclear accumulation of FoxO3 [6]. FoxO3 had been verified as a key protein in the suppression of cancer progression, with roles including control of differentiation and tumorigenicity through the PI3K/Akt/mTOR and MEK/ ERK signaling pathways [55-57]. Nuclear accumulation of FOXO3a could be promoted by miR-22 and was observed to subsequently reverse invasive phenotype of HCC cells through repression of YWHAZ-mediated AKT phosphorylation [10]. Besides, the expression of YWHAZ could be negatively regulated by miR-30c in cervical cancer, by $m i R-544$ in breast cancer, and by miR-613 in HCC [58-60].

Recently, long non-coding RNAs, more than 200nt and involved in multiple cell processes, are emerging as competing endogenous RNAs to regulate YWHAZ by targeting miRNAs [61-63]. In gastric cancer, long non-coding RNA LUCAT1 was negatively correlated with $m i R-134-5 p$ and $m i R-134-5 p$ 
was negatively related with YWHAZ [61]. Knockdown of LUCAT1 inhibited YWHAZ expression, which can be reversed by miR-134-5p inhibitor [61]. Similarly, long non-coding RNA SNHG14, acting as a miR-206 sponge and decreasing its expression, increased YWHAZ expression in cervical cancer [62] and long non-coding RNA LINC00858 regulated YWHAZ by inhibiting miR-22-3p in colorectal cancer [63].

\section{Downstream targets of YWHAZ}

\section{YWHAZ and protein phosphorylation}

The 14-3-3 family interacted with a diverse range of cell signaling proteins by binding to an amphipathic helix and activating it through phosphorylation $[64,65]$. Doubly phosphorylated peptides tightly bound simultaneously at adjacent 14-3-3 sites to form high-affinity bidentate complexes [65]. YWHAZ was found to play an important role in chemoresistance through modulation of protein phosphorylation. In HCC, aB-Crystallin-YWHAZ complexes were observed to promote EMT through elevated ERK1/2 phosphorylation, which impaired the effect of sorafenib, while JNK and p38/MAPK phosphorylation were verified to increase chemosensitivity of HCC cells to CDDP when YWHAZ was silenced [11, 14]. Moreover, Cdc2, belonging to the cyclindependent kinase family, is a maturation-promoting factor involving in the G2-M transition [66]. Cdc2 phosphorylation had been observed after YWHAZ reduction, subsequently sensitizing lung cancer cells to cisplatin-induced G2-M arrest [67].

\section{YWHAZ and apoptosis protein}

Pro-apoptotic proteins Caspase- 3 and Bax were increased in YWHAZ-depleted liver cancer stem-like cells (CSCs) [13]. Conversely, a dramatic loss of Bax and caspase- 3 were observed in breast cancer cells overexpressing YWHAZ [38]. Neal CL et al. also found that decreased YWHAZ sensitized breast cancer cells to apoptosis in low serum conditions by increasing cytochrome C release, subsequently reducing procaspase 9 expression and caspase substrate cleavage [32]. These studies demonstrated that YWHAZ may induce apoptosis resistance by modulating mitochondrial apoptosis pathways.

\section{YWHAZ and metastasis-related molecules}

ErbB2, a receptor tyrosine-protein kinase, was overexpressed in approximately $20 \%-30 \%$ of BRCA and played a vital role in the development and metastasis of BRCA [68, 69]. Co-overexpression of YWHAZ and ErbB2 in ductal CIS conferred an increased risk of progression to invasive BRCA than those overexpressed one molecule alone [39]. This was believed to occur through activation of the TGF-b/Smads pathway, which subsequently led to ZFHX1B/SIP-1 up-regulation, E-cadherin loss, and EMT [39]. Kambach DM et al. also demonstrated that ionizing radiation-induced YWHAZ upregulation was required and sufficient for cell invasion in ErbB2-positive BRCA cells, together with FoxM1 [40]. Transforming growth factor- $\beta$ (TGF- $\beta$ ) functions as a tumor suppressor in premalignant cells but, interestingly, as a metastasis promoter in cancer cells [70]. In breast cancer cells, YWHAZ destabilized $p 53$ and stabilizes Gli2, promoting TGF- $\beta$-induced bone metastasis [70]. Binding of Axl to YWHAZ caused Smad3L phosphorylation and then resulted in the up-regulation of TGF- $\beta$ target genes and TGF- $\beta 1$ in mesenchymal HCC cells, which is essentially required for Axl-mediated cell invasion [15]. Under hypoxia condition, HIF-1a could be induced, acting as a crucial factor for tumor metastasis in HCC [71]. YWHAZ enhanced HIF-1a protein stability and recruited HDCA4 to inhibit HIF-1a acetylation, subsequently promoting HCC cell metastasis via HIF-1a/EMT or PI3K/Akt/NF-KB signaling pathway $[12,71]$.

Taken together, some crucial upstream regulators and downstream targets of YWHAZ involving in cancer progression were summarized in Table 1. Importantly, three HCC RNA-seq datasets (GSE69164, GSE63863, and GSE55758) from Gene Expression Omnibus (GEO) indicated that $Y W H A Z$ is a hub gene in HCC [72]. Hence, we summarized the verified signaling networks of YWHAZ in HCC to systematically understand its role [5, 10, 12, 14, 15] (Figure 2).

\section{YWHAZ as a potential biomarker in cancer}

\section{Diagnosis}

In the past decades, the diagnostic potential of YWHAZ had aroused considerable interest. Liu M et al. detected that the prevalence of YWHAZ autoantibodies was $16.7 \%(28 / 168)$ in HCC, significantly higher than in liver cirrhosis, chronic hepatitis, and normal human sera by enzyme-linked immunosorbent assay (ELISA) analysis $(\mathrm{P}<0.01)$ [73]. Similarly, ELISA showed that autoantibody to YWHAZ was obviously higher in 465 gastric cancer patients $(0.17 \pm$ $0.08 \mathrm{ng} / \mathrm{ml})$ compared to 465 normal samples $(0.14 \pm$ 0.06) $(\mathrm{P}<0.001)$ [74]. Moreover, $\mathrm{YWHAZ}$ autoantibody combined with diagnosis biomarkers of gastric cancer (CEA, CA199, CA724), increasing the diagnostic sensitivity to $52.7 \%$ [74]. Zhang $\mathrm{Y}$ et al. discovered that YWHAZ, as well as HTR2B, CHL1, the ZNF family and $F Y N$, were observed to be most obviously 
altered between 46 liver metastatic uveal melanoma samples and 45 non-metastatic uveal melanoma samples, derived from GEO database [75]. This distinction may provide diagnostic and preventative worth for uveal melanoma liver metastases in the future [75]. Huang Y-D et al. identified genes related to bladder cancer using microarray chip, detecting that YWHAZ, PRDX2 and C1QBP were all related to inflammation and cell proliferation and could be regarded as candidate biomarkers for bladder cancer diagnosis [76]. In conclusion, these studies demonstrated that YWHAZ or combination of YWHAZ and clinical markers may be promising diagnostic biomarker in the future.

\section{Prognosis}

Our team used Kaplan-Meier survival analysis to explore the relationship between YWHAZ expression and overall survival/disease-free survival at 60 months in BRCA, COAD, ESCA, LIHC, LUAD, LUSC, PRAD, READ, and STAD from the Cancer Genome Atlas (TCGA) database. As can be seen in Figure 3A and Figure 4A, YWHAZ expression was significantly correlated with overall survival at 60 months in LIHC $(p=0.0197)$ and LUAD $(p=0.016)$, and with disease-free survival at 60 months in BRCA $(p=0.0279)$ and LUAD $(p=0.016)$. We also conducted Kaplan-Meier survival analysis in Gene Expression Profiling Interactive Analysis (GEPIA) database with larger samples [77], determining that overall survival time was remarkably longer in LIHC $(p=0.016)$ and LUAD ( $\mathrm{p}=0.00023$ ) with low YWHAZ expression (Figure 3B) [24], which was consistent with the result of TCGA. However, there was no statistic difference of disease-free survival between high YWHAZ and low YWHAZ samples (Figure 4B).

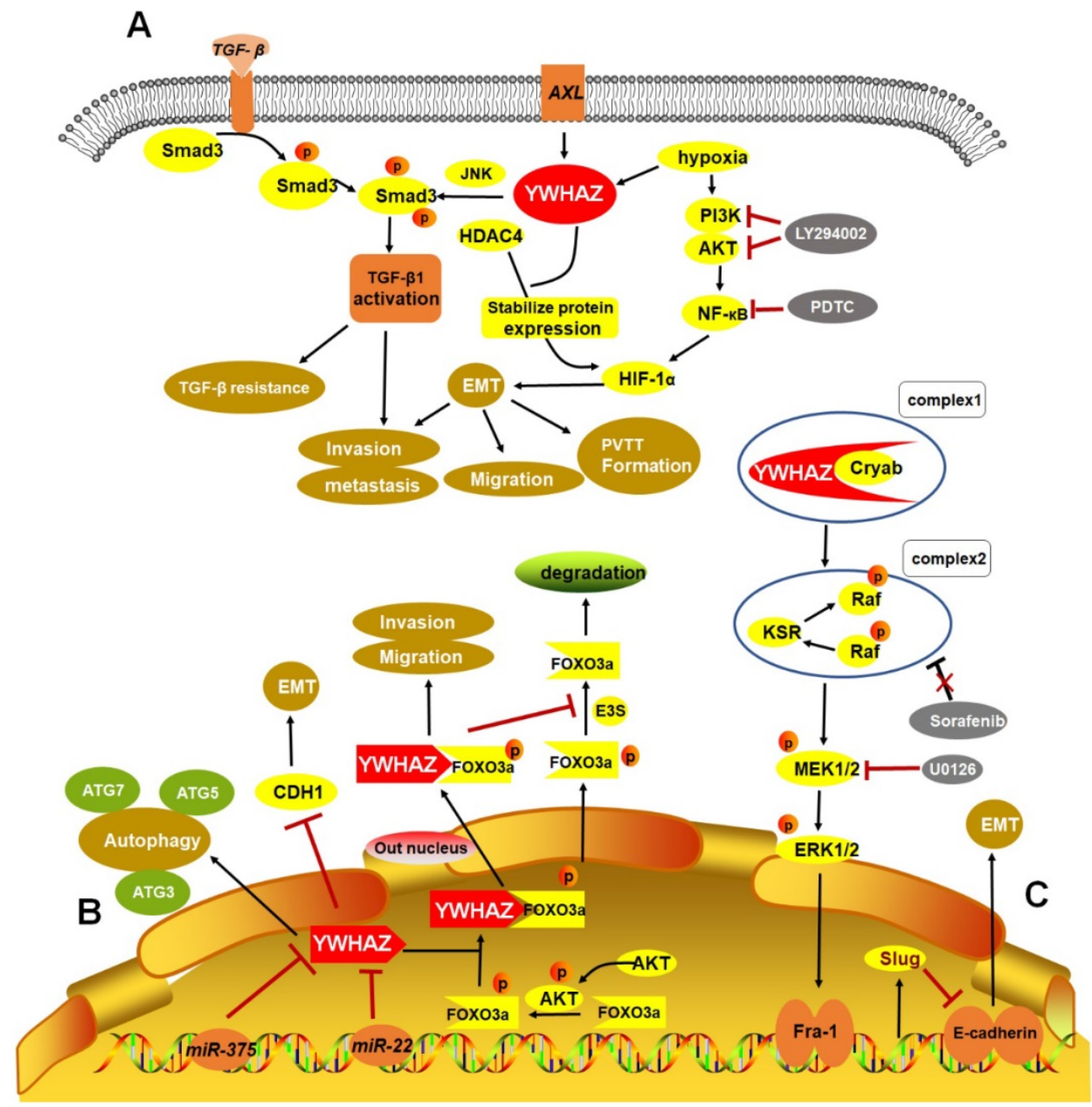

Figure 2. Verified signaling pathways of YWHAZ in HCC. A. Phosphorylation of Smad3 linker region by AxI/YWHAZ and JNK activates the expression of TGF- $\beta$ I, leading to HCC invasion and metastasis by TGF- $\beta$ resistance. Additionally, under hypoxic circumstance, YWHAZ interacts with HIF-1 $\alpha$ and enhances HIF-1 $\alpha$ protein stability by recruiting HDAC4 and activating PI3K/Akt/NF-KB pathway, then inducing cell migration, invasion and PVTT formation in HCC. B. MiR-375, miR-22 directly targets 3'-UTR of YWHAZ mRNA to inhibit YWHAZ expression. YWHAZ can induce autophagy by ATG3, ATG5, ATG7 and promote EMT by suppressing CDH1 in HCC. Additionally, phosphorylated AKT inhibits the activity of FOXO3a by promoting its phosphorylation and binding YWHAZ with FOXO3a, then promoting HCC migration and invasion. In cytoplasm, YWHAZ/FOXO3a complex inhibits the dephosphorylation of phosphorylated FOXO3a and promotes FOXO3a degradation. C. Cryab complexes with YWHAZ and elevates its expression, leading to activation of ERK1/2/Fra-1/slug signaling pathway and then inducing EMT progression by decreasing E-cadherin expression. Moreover, sorafenib response is impaired in this signaling pathway. HCC: hepatocellular carcinoma; TGF- $\beta$ : transforming growth factor- $\beta$; HIF-1 $\alpha$ : hypoxia-induced factor-1 $\alpha$; EMT: epithelial-mesenchymal transition; PVTT: portal vein tumor thrombus; Cryab: $\alpha B-C r y s t a l l i n$. 
A

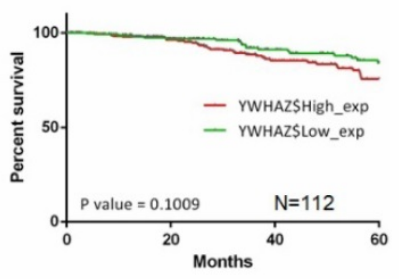

LIHC

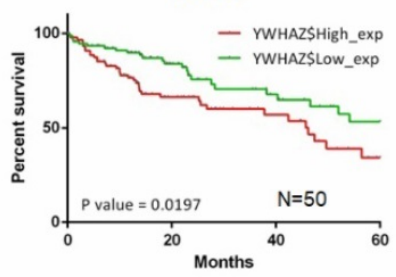

PRAD

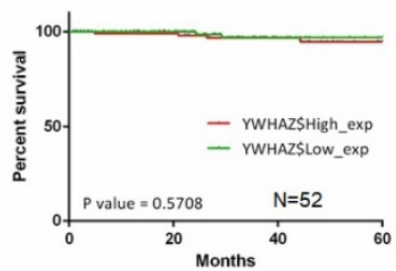

B
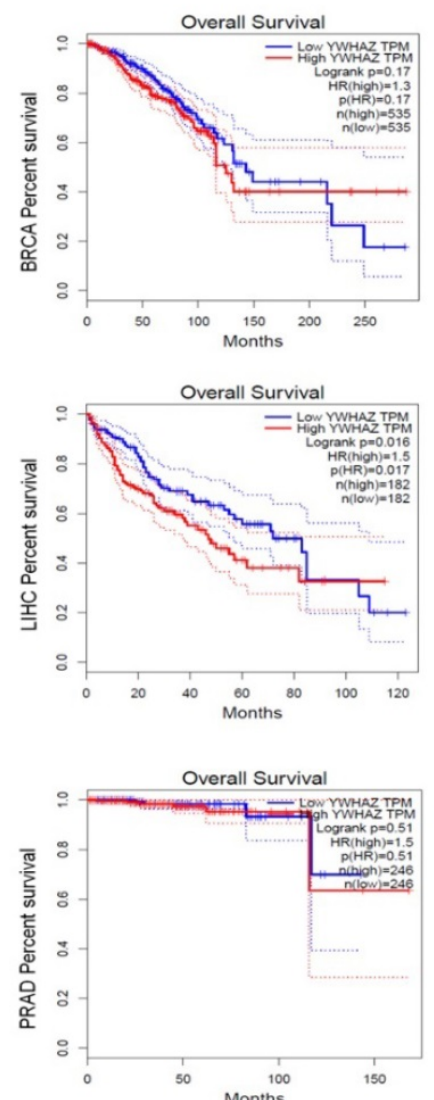

COAD

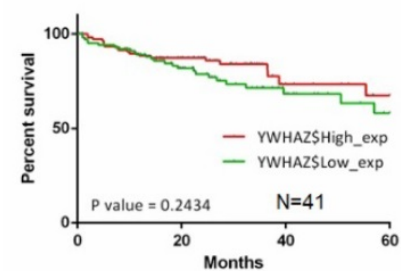

LUAD

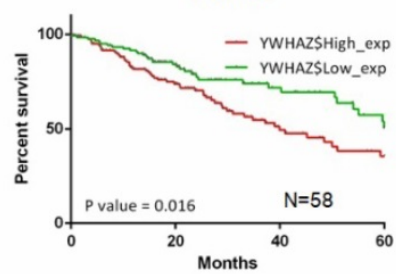

READ
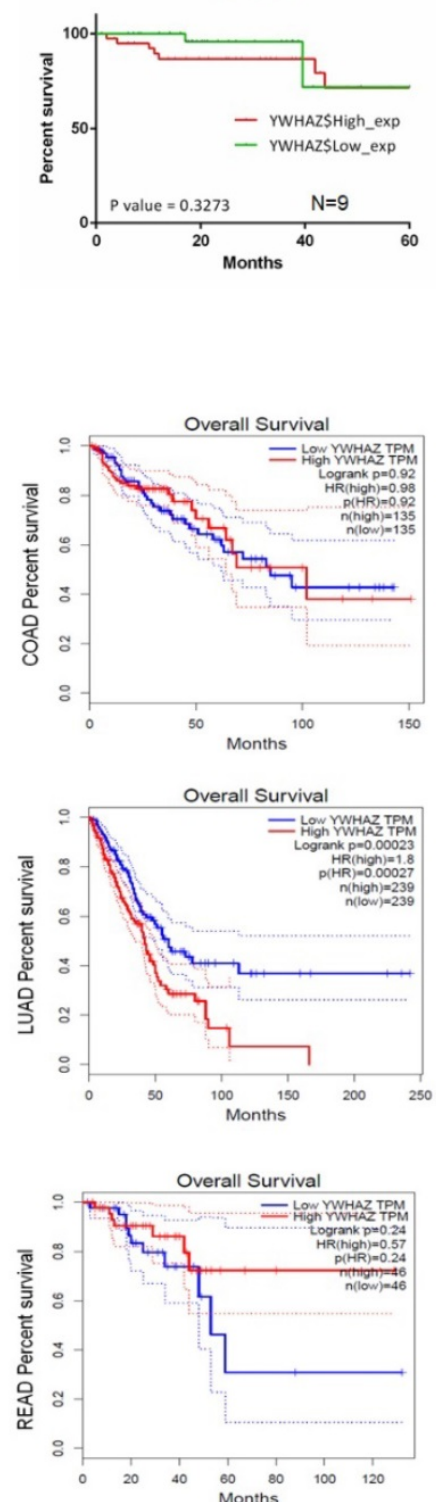

ESCA

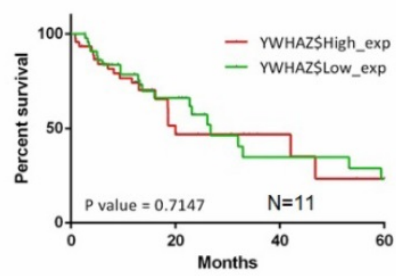

LUSC

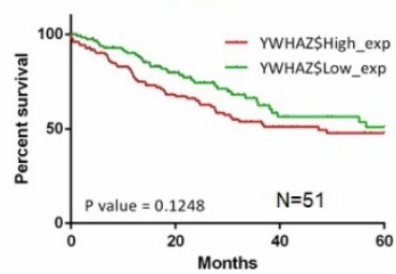

STAD
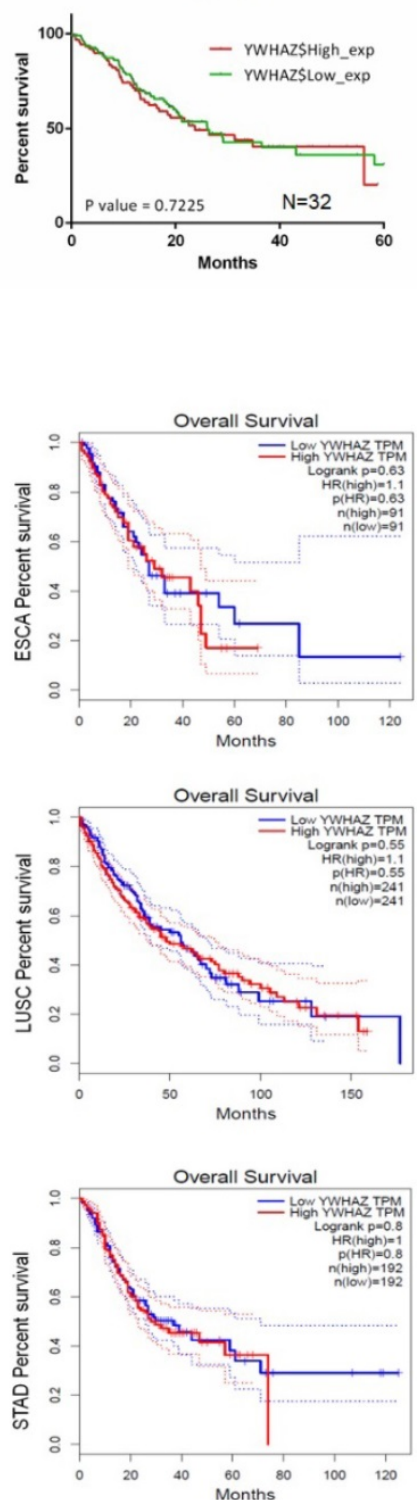

Figure 3. The correlation between YWHAZ expression and overall survival in multiple types of cancers. The $x$ axis is the overall survival month, and the $y$ axis represents the survival rate. A. Kaplan-Meier survival analysis of YWHAZ at 60 months is shown. These data were derived from TCGA database. Group cutoff is quartile. B. Overall survival analysis of YWHAZ from GEPIA database. Group cutoff is median. Abbreviations are as marked in Figure 1. 
A

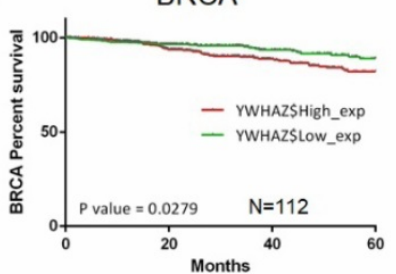

LIHC
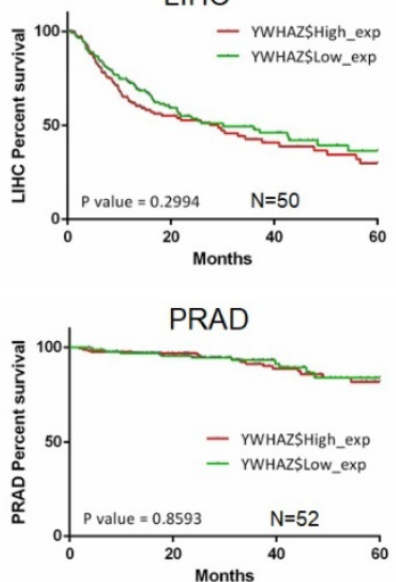

B
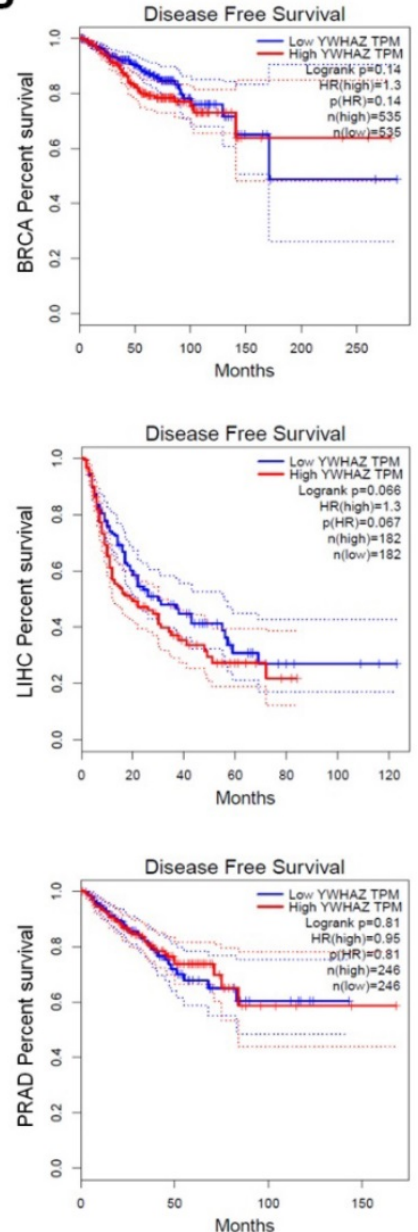
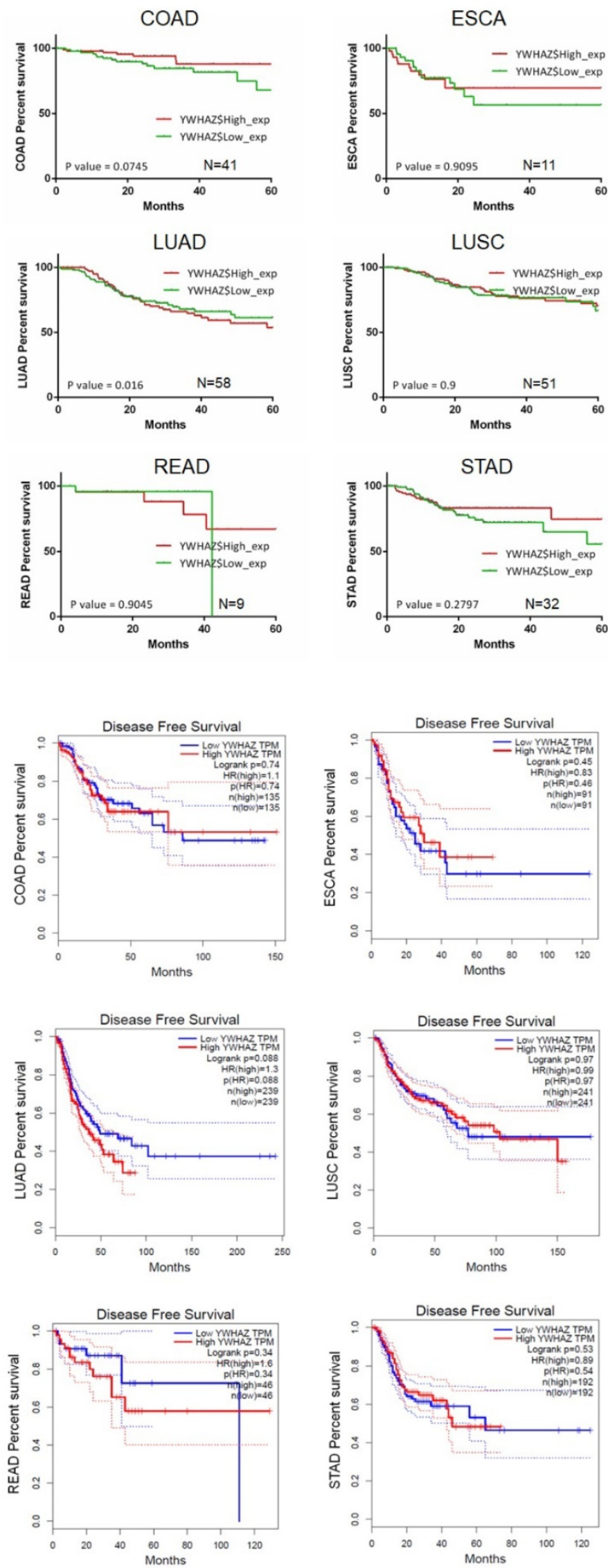

Figure 4. The correlation between YWHAZ expression and disease-free survival in multiple types of cancer. The $\mathrm{x}$ axis is the disease-free survival month, and the $y$ axis represents the survival rate. A. Kaplan-Meier survival analysis of YWHAZ at 60 months is shown. These data were derived from TCGA. Group cutoff is median. B. Disease-free survival analysis of YWHAZ from GEPIA database. Group cutoff is median. Abbreviations are as marked in Figure 1. 
In HCC, we observed that combination of ASH1, miR-375 and YWHAZ resulted in significant differences regarding overall survival at 50 months $(p$ $=0.003), 60$ months $(p=0.0096)$ and 100 months $(p=$ 0.0158 ) [5]. Yufu T et al. demonstrated differences ( $\mathrm{P}<$ 0.001 ) in both overall survival ( 28 vs. $>33$ months) and time to recurrence (12 vs. 24 months) between high HIF-1a/YWHAZ vs. low HIF-1a/ YWHAZ HCC groups [12]. Furthermore, decreased survival ( $P$ $=0.025)$ was also considered to be strongly associated with elevated levels of YWHAZ and Axl in HCC [15]. Fan $T$ et al. reported that overall survival at 5 years after surgery and cancer-specific survival in stage I NSCLC YWHAZ-positive patients were 0.36 and 0.60 , compared with 0.68 and 0.95 in YWHAZ-negative patients [67]. LUSC patients with high YWHAZ/ TGF $\beta$ receptor types 1 (TGF $\beta R 1$ ) have shorter overall survival than patients with low YWHAZ/TGF $\beta R 1$ [27]. Similarly, YWHAZ overexpression was significantly associated with reduced disease-free survival/overall survival and earlier time to disease recurrence, and death in breast cancer by combining with elevated levels of Akt, FOXM1, ErbB2, LOC441453 and LAPTM4B [31, 32, 35, 36, 39, 78-80]. In particular, YWHAZ overexpression, ErbB2 overexpression, and positive lymph node status were seen to be independent prognostic factors in breast cancer [39]. In head-and-neck/oral squamous cell carcinoma, disease-free survival of the YWHAZ-positive group was 23 months compared with 35 months for the YWHAZ-negative group [81]. In glioblastoma, 2-year overall survival and median survival time in the YWHAZ-positive group were $8.6 \%$ and 12.9 months, compared with $16.7 \%$ and 17.9 months in the YWHAZ-negative group [82]. Furthermore, a growing number of studies have proposed that elevated YWHAZ expression was correlated with poor prognosis in prostate cancer [44], ICC [48], and gastric carcinoma [21], implying that YWHAZ was tightly associated with the survival of cancer patients.

\section{Chemoresistance}

It is well known that barriers to chemotherapeutic agents during cancer therapy include intrinsic and acquired resistance, thus the effect of chemotherapy among cancer patients is still often sub-optimal. The anti-apoptosis ability exerted by YWHAZ may be responsible for chemoresistance. High levels of YWHAZ had been found in CHOP-resistant DLBCL cells and 9-nitrocamptothecin resistant prostate cancer cells, compared with chemo-sensitive cells $[49,83]$. Intriguingly, YWHAZ knockdown had been shown to restore the sensitivity of resistant cells to apoptosis induced by chemotherapeutic agents including $\mathrm{CHOP}$, 9-nitrocamptothecin,
CDDP, cisplatin and TPT $[11,47,49,67,83]$. In breast cancer, silencing of LAPTM4B and YWHAZ gene sensitized tumor cells to anthracyclines, while overexpression of these genes induced drug resistance [79]. Moreover, YWHAZ knockdown enhanced the growth inhibitory effects of SERMs in endocrineresistant breast cancer cells, restoring sensitivity to endocrine treatments $[35,54]$. Based on the above evidence, it is promising to target YWHAZ to decrease chemoresistance and improve the effect of chemotherapy.

\section{Therapeutic potential}

Surgery, chemotherapy and radiotherapy have traditionally been the main therapeutic methods for human cancers. However, the prognosis of most cancer patients treated through these approaches still remain fairly poor. Given the oncogene role of YWHAZ in multiple cancers, the combination of traditional therapeutic methods and YWHAZtargeted therapies may be an attractive project in the future.

Our team delivered si-NC, si-YWHAZ and si-YWHAZ/DOX using nanoliposomes (L) in established mouse HCC xenograft models, observing that tumor growth could be inhibited in the latter two groups compared with control [5]. IHC analysis further revealed that cell proliferation was inhibited and cell apoptosis was increased in vivo by YWHAZ blockade [5]. Neal CL et al. observed delayed breast cancer onset and reduced tumor growth in mice injected with YWHAZ siRNA using lipofectamine [32]. Similarly, nude mice were inoculated with lung cancer cells, shRNA-control lung cancer cells and sh-YWHAZ lung cancer cells using lipofectamine [67]. Results of the three groups showed that tumor volumes were $169.49 \pm 20.61,154.54 \pm 20.06$, and $151.49 \pm 34.78 \mathrm{~mm}^{3}$ after 17 days $(\mathrm{P}=0.091)$ and tumor growth ratios were $54 \%, 50 \%$ and $22 \%$ by 28 days after the initiation of cisplatin treatment, implying a suppressive role of YWHAZ knockdown [67]. Yufu T et al. used HCC-CSQT-2/sh-YWHAZ cells, which are derived from PVTT and prone to form PVTT, to establish orthotopic transplantation assays in nude mice [12]. Using these techniques, they established mouse models of PVTT by injecting HCC-CSQT-2/ sh-YWHAZ cells or HCC- CSQT-2/sh-control cells into mice through the tail vein [12]. Results from this study indicated that blockade of YWHAZ by shRNA suppressed lung metastases and formation of PVTT in vivo [12]. Therapeutic approaches which increase expression of microRNAs targeting $Y W H A Z$ might also be worth exploring. Up-regulation of miRNA-451 by murine stem cell virus vector directly decreased YWHAZ expression and inhibited colon cancer 
growth in vitro and in vivo [6]. However, compensatory effects of siRNA, shRNA or miRNA approach by targeting a single molecule do exist after long term treatment. Recently, proteolysis targeting chimera (PROTAC) technology has attracted people's interest for its promise in disease therapeutics that induced targeted protein degradation and has made success in a selective small-molecule degrader of STAT3 which achieved complete tumor regression in vivo [84]. Thus, chemical compound or molecular inhibitors targeting $\mathrm{YWHAZ}$ specifically are greatly needed in the future.

\section{Conclusion and future direction}

To date, YWHAZ has been shown to be frequently up-regulated and function as an oncogene by regulating multiple signaling pathways in cancers (Table 1). YWHAZ overexpression is regulated by miRNAs or long non-coding RNAs and activates downstream molecules, including protein kinases, apoptosis proteins, and metastasis-related molecules, to facilitate the malignant potential of cancer cells. However, a comprehensive assessment of YWHAZ regulatory networks through bioinformatics analysis is warranted. Growing evidences suggested the potential role of YWHAZ in cancer diagnosis, prognosis and chemoresistance. However the specificity and sensibility of YWHAZ as an independent biomarker are limited. Combinations of YWHAZ with other cancer-specific molecules may have better ability to serve as biomarkers. At present, YWHAZ targeting therapy alone through siRNA, shRNA or miRNA to delay tumor development shows some preliminary results. Nevertheless, safer and more effective carriers for YWHAZ inhibitor delivery, or combinations of YWHAZ with other promising therapeutic targets are greatly needed. In summary, YWHAZ, acting as an important oncogene, is increasingly showing its potential as a biomarker for diagnosis, prognosis, chemoresistance and therapeutic target in a diverse range of malignancies.

\section{Acknowledgements}

This work was financially supported by the National Natural Science Foundation of China (No. 81772969), and the Applied Basic Research Program of Wuhan City (No. 2017060201010155).

\section{Competing Interests}

The authors have declared that no competing interest exists.

\section{References}

1. Jones DH, Ley S, Aitken A. Isoforms of 14-3-3 protein can form homoand heterodimers in vivo and in vitro: implications for function as adapter proteins. Febs Letters. 1995; 368: 55.
2. Qi W, Liu X, Qiao D, et al. Isoform-specific expression of 14-3-3 proteins in human lung cancer tissues. Int J Cancer. 2005; 113: 359-63.

3. Liang S, Shen G, Liu Q, et al. Isoform-specific expression and characterization of 14-3-3 proteins in human glioma tissues discovered by stable isotope labeling with amino acids in cell culture-based proteomic analysis. Proteomics Clin Appl. 2009; 3: 743-53.

4. Aitken A, Howell S, Jones D, et al. 14-3-3 a and $\delta$ Are the Phosphorylated Forms of Raf-activating 14-3-3 $\beta$ and $\zeta$. J Biol Chem. 1995; 270: 5706-9.

5. Zhao JF, Zhao Q, Hu H, et al. The ASH1-miR-375-YWHAZ Signaling Axis Regulates Tumor Properties in Hepatocellular Carcinoma. Mol Ther Nucleic Acids. 2018; 11: 538-53.

6. Li Y, Wang J, Dai X, et al. miR-451 regulates FoxO3 nuclear accumulation through Ywhaz in human colorectal cancer. Am J Transl Res. 2015; 7: 2775-85.

7. Tong S, Xia T, Fan K, et al. 14-3-3zeta promotes lung cancer cell invasion by increasing the Snail protein expression through atypical protein kinase C (aPKC)/NF-kappaB signaling. Exp Cell Res. 2016; 348: 1-9.

8. Wang $\mathrm{W}$, Zhang $\mathrm{L}$, Wang $\mathrm{Y}$, et al. Involvement of miR-451 in resistance to paclitaxel by regulating YWHAZ in breast cancer. Cell Death Dis. 2017; 8: e3071.

9. Chandrashekar DS, Bashel B, Balasubramanya SAH, et al. UALCAN: A Portal for Facilitating Tumor Subgroup Gene Expression and Survival Analyses. Neoplasia. 2017; 19: 649-58.

10. Chen $\mathrm{M}, \mathrm{Hu} \mathrm{W}$, Xiong $\mathrm{CL}$, et al. miR-22 targets $\mathrm{YWHAZ}$ to inhibit metastasis of hepatocellular carcinoma and its down-regulation predicts a poor survival. Oncotarget. 2016; 7: 80751-64.

11. Choi JE, Hur W, Jung CK, et al. Silencing of 14-3-3zeta over-expression in hepatocellular carcinoma inhibits tumor growth and enhances chemosensitivity to cis-diammined dichloridoplatium. Cancer Lett. 2011; 303: 99-107.

12. Tang $\mathrm{Y}, \mathrm{Liu} \mathrm{S}, \mathrm{Li} \mathrm{N}$, et al. $14-3-3 \zeta$ promotes hepatocellular carcinoma venous metastasis by modulating hypoxia-inducible factor-1a. Oncotarget. 2016; 7: 15854-67.

13. Lee YK, Hur W, Lee SW, et al. Knockdown of 14-3-3zeta enhances radiosensitivity and radio-induced apoptosis in CD133(+) liver cancer stem cells. Exp Mol Med. 2014; 46: e77.

14. Huang XY, Ke AW, Shi GM, et al. alphaB-crystallin complexes with 14-3-3zeta to induce epithelial-mesenchymal transition and resistance to sorafenib in hepatocellular carcinoma. Hepatology. 2013; 57: 2235-47.

15. Reichl P, Dengler M, Van ZF, et al. Axl activates autocrine transforming growth factor- $\beta$ signaling in hepatocellular carcinoma. Hepatology. 2015; 61: 930-41.

16. Song J, Zhang X, Liao Z, et al. 14-3-3zeta inhibits heme oxygenase- 1 (HO-1) degradation and promotes hepatocellular carcinoma proliferation: involvement of STAT3 signaling. J Exp Clin Cancer Res. 2019; 38: 3 .

17. Mu Y, Chen Y, Zhang G, et al. Identification of stromal differentially expressed proteins in the colon carcinoma by quantitative proteomics. Electrophoresis. 2013; 34: 1679-92.

18. Mulvey HE, Chang A, Adler J, et al. Extracellular vesicle-mediated phenotype switching in malignant and non-malignant colon cells. BMC Cancer. 2015; 15: 571.

19. Sheng N, Yan L, Wu K, et al. TRIP13 promotes tumor growth and is associated with poor prognosis in colorectal cancer. Cell Death Dis. 2018; 9: 402.

20. Guo F, Gao Y, Sui G, et al. miR-375-3p/YWHAZ/ $\beta$-catenin axis regulates migration, invasion, EMT in gastric cancer cells. Clin Exp Pharmacol Physiol. 2019; 46: 144-52.

21. Nishimura Y, Komatsu S, Ichikawa D, et al. Overexpression of YWHAZ relates to tumor cell proliferation and malignant outcome of gastric carcinoma. Br J Cancer. 2013; 108: 1324-31.

22. Tsukamoto Y, Nakada C, Noguchi T, et al. MicroRNA-375 is downregulated in gastric carcinomas and regulates cell survival by targeting PDK1 and 14-3-3zeta. Cancer Res. 2010; 70: 2339-49.

23. Guo F, Jiao D, Sui GQ, et al. Anticancer effect of YWHAZ silencing via inducing apoptosis and autophagy in gastric cancer cells. Neoplasma. 2018; 65: 693-700

24. Deng Y, Zheng J, Ma J. The clinical and prognostic significance of YWHAZ in non-small-cell lung cancer patients: Immunohistochemical analysis. J Cell Biochem. 2019; 120: 6290-8.

25. Zhao G-Y, Ding J-Y, Gu J, et al. The overexpression of 14-3-3 $\zeta$ and Hsp27 promotes non-small cell lung cancer progression. Cancer. 2014; 120: 652-63.

26. Chen $\mathrm{CH}$, Chuang $\mathrm{SM}$, Yang $\mathrm{MF}$, et al. A novel function of YWHAZ/ $\beta$-catenin axis in promoting epithelial-mesenchymal transition and lung cancer metastasis. Mol Cancer Res. 2012; 10: 1319-31.

27. Zhao Y, Qiao W, Wang X, et al. 14-3-3zeta/TGFbetaR1 promotes tumor metastasis in lung squamous cell carcinoma. Oncotarget. 2016; 7: 82972-84 
28. Li Z, Zhao J, Du Y, et al. Down-regulation of 14-3-3zeta suppresses anchorage-independent growth of lung cancer cells through anoikis activation. Proc Natl Acad Sci U S A. 2008; 105: 162-7.

29. Xue M, Tao W. Upregulation of MUC1 by its novel activator 14-3-3zeta promotes tumor invasion and indicates poor prognosis in lung adenocarcinoma. Oncol Rep. 2017; 38: 2637-46.

30. Song T, Tian X, Kai F, et al. Loss of Par3 promotes lung adenocarcinoma metastasis through 14-3-3zeta protein. Oncotarget. 2016; 7: 64260-73.

31. Bergamaschi A, Frasor J, Borgen K, et al. 14-3-3zeta as a predictor of early time to recurrence and distant metastasis in hormone receptor-positive and -negative breast cancers. Breast Cancer Res Treat. 2013; 137: 689-96.

32. Neal CL, Yao J, Yang W, et al. 14-3-3zeta overexpression defines high risk for breast cancer recurrence and promotes cancer cell survival. Cancer Res. 2009; 69: 3425-32.

33. Liu ZR, Song Y, Wan LH, et al. Over-expression of miR-451a can enhance the sensitivity of breast cancer cells to tamoxifen by regulating 14-3-3zeta, estrogen receptor alpha, and autophagy. Life Sci. 2016; 149: 104-13.

34. Leivonen SK, Rokka A, Ostling P, et al. Identification of miR-193b targets in breast cancer cells and systems biological analysis of their functional impact. Mol Cell Proteomics. 2011; 10: M110.005322.

35. Bergamaschi A, Christensen BL, Katzenellenbogen BS. Reversal of endocrine resistance in breast cancer: interrelationships among 14-3-3zeta, FOXM1, and a gene signature associated with mitosis. Breast Cancer Res. 2011; 13: R70.

36. Neal CL, Xu J, Li P, et al. Overexpression of 14-3-3zeta in cancer cells activates PI3K via binding the p85 regulatory subunit. Oncogene. 2012; 31: 897-906.

37. Rehman SK, Li SH, Wyszomierski SL, et al. 14-3-3zeta orchestrates mammary tumor onset and progression via miR-221-mediated cell proliferation. Cancer Res. 2014; 74: 363-73.

38. Danes CG, Wyszomierski SL, Lu J, et al. 14-3-3 zeta down-regulates p53 in mammary epithelial cells and confers luminal filling. Cancer Res. 2008; 68: 1760-7.

39. Lu J, Guo H, Treekitkarnmongkol W, et al. 14-3-3zeta Cooperates with ErbB2 to promote ductal carcinoma in situ progression to invasive breast cancer by inducing epithelial-mesenchymal transition. Cancer Cell. 2009; 16: 195-207.

40. Kambach DM, Sodi VL, Lelkes PI, et al. ErbB2, FoxM1 and 14-3-3zeta prime breast cancer cells for invasion in response to ionizing radiation. Oncogene. 2014; 33: 589-98.

41. Taro M, Ken-Ichi T, Tomohiko U, et al. 14-3-3ろ, a novel androgen-responsive gene, is upregulated in prostate cancer and promotes prostate cancer cell proliferation and survival. Clin Cancer Res. 2012; 18: 5617-27.

42. Goc A, Abdalla M, Al-Azayzih A, et al. Rac1 activation driven by 14-3-3zeta dimerization promotes prostate cancer cell-matrix interactions, motility and transendothelial migration. PLoS One. 2012; 7: e40594.

43. Menon R, Deng M, Ruenauver $K$, et al. Somatic copy number alterations by whole-exome sequencing implicates YWHAZ and PTK2 in castration-resistant prostate cancer. J Pathol. 2013; 231: 505-16.

44. Ruenauver K, Menon R, Svensson MA, et al. Prognostic significance of YWHAZ expression in localized prostate cancer. Prostate Cancer Prostatic Dis. 2014; 17: 310-4.

45. Tzivion G, Luo Z, Avruch J. A dimeric 14-3-3 protein is an essential cofactor for Raf kinase activity. Nature. 1998; 394: 88-92.

46. Su R, Gong JN, Chen MT, et al. c-Myc suppresses miR-451 dash, verticalYWTAZ/AKT axis via recruiting HDAC3 in acute myeloid leukemia. Oncotarget. 2016; 7: 77430-43.

47. Liang $\mathrm{R}$, Chen $\mathrm{XQ}$, Bai QX, et al. Increased 14-3-3zeta expression in the multidrug-resistant leukemia cell line HL-60/VCR as compared to the parental line mediates cell growth and apoptosis in part through modification of gene expression. Acta Haematol. 2014; 132: 177-86.

48. Zhang C, Liu LX, Dong ZR, et al. Up-regulation of 14-3-3zeta expression in intrahepatic cholangiocarcinoma and its clinical implications. Tumour Biol. 2015; 36: 1781-9.

49. Maxwell SA, Li Z, Jaye D, et al. 14-3-3zeta mediates resistance of diffuse large B cell lymphoma to an anthracycline-based chemotherapeutic regimen. J Biol Chem. 2009; 284: 22379-89.

50. Chen K, Rajewsky N. The evolution of gene regulation by transcription factors and microRNAs. Nat Rev Genet. 2007; 8: 93-103.

51. Calin GA, Croce CM. MicroRNA signatures in human cancers. Nat Rev Cancer. 2006; 6: 857-66.

52. Di Leva G, Croce CM. miRNA profiling of cancer. Curr Opin Genet Dev. 2013; 23: 3-11.

53. Jung HM, Phillips BL, Chan EK. miR-375 activates p21 and suppresses telomerase activity by coordinately regulating HPV E6/E7, E6AP, CIP2A, and 14-3-3zeta. Mol Cancer. 2014; 13: 80.
54. Bergamaschi A, Katzenellenbogen BS. Tamoxifen downregulation of miR-451 increases 14-3-3zeta and promotes breast cancer cell survival and endocrine resistance. Oncogene. 2012; 31: 39-47.

55. Lin C, Wu Z, Lin X, et al. Knockdown of FLOT1 Impairs Cell Proliferation and Tumorigenicity in Breast Cancer through Upregulation of FOXO3a. Clin Cancer Res. 2011; 17: 3089-99.

56. Sunayama J, Sato A, Matsuda K, et al. FoxO3a functions as a key integrator of cellular signals that control glioblastoma stem-like cell differentiation and tumorigenicity. Stem Cells. 2011; 29: 1327-37.

57. Yang W, Dolloff NG, El-Deiry WS. ERK and MDM2 prey on FOXO3a. Nat Cell Biol. 2008; 10: 125-6.

58. Fang $\mathrm{Y}$, Shen $\mathrm{H}, \mathrm{Cao} \mathrm{Y}$, et al. Involvement of miR-30c in resistance to doxorubicin by regulating YWHAZ in breast cancer cells. Braz J Med Biol Res. 2014; 47: 60-9.

59. Mao L, Yan Z, Deng X, et al. Transcription factor KLF4 regulates microRNA-544 that targets YWHAZ in cervical cancer. Am J Cancer Res. 2015; 5: 1939-53.

60. Jiang $\mathrm{X}, \mathrm{Wu}$ J, Zhang $\mathrm{Y}$, et al. MiR-613 functions as tumor suppressor in hepatocellular carcinoma by targeting YWHAZ. Gene. 2018; 659: 168-74.

61. Chi J, Liu T, Shi C, et al. Long non-coding RNA LUCAT1 promotes proliferation and invasion in gastric cancer by regulating miR-134-5p/YWHAZ axis. Biomed Pharmacother. 2019; 118: 109201.

62. Ji N, Wang Y, Bao G, et al. LncRNA SNHG14 promotes the progression of cervical cancer by regulating miR-206/YWHAZ. Pathol Res Pract. 2019; 215: 668-75.

63. Sha QK, Chen L, Xi JZ, et al. Long non-coding RNA LINC00858 promotes cells proliferation, migration and invasion by acting as a ceRNA of miR-22-3p in colorectal cancer. Artif Cells Nanomed Biotechnol. 2019; 47: 1057-66.

64. Muslin AJ, Tanner JW, Allen PM, et al. Interaction of 14-3-3 with Signaling Proteins Is Mediated by the Recognition of Phosphoserine. Cell. 1996; 84: 889-97.

65. Mackintosh C. Dynamic interactions between 14-3-3 proteins and phosphoproteins regulate diverse cellular processes. Biochem J. 2004; 381: 329-42.

66. Stark GR, Taylor WR. Analyzing the G2/M Checkpoint. Methods Mol Biol. 2004; 280: 51-82.

67. Fan T, Li R, Todd NW, et al. Up-regulation of 14-3-3zeta in lung cancer and its implication as prognostic and therapeutic target. Cancer Res. 2007; 67: 7901-6.

68. Yu D, Hung MC. Overexpression of ErbB2 in cancer and ErbB2-targeting strategies. Oncogene. 2000; 19: 6115-21.

69. Mitri Z. The HER2 Receptor in Breast Cancer: Pathophysiology, Clinical Use, and New Advances in Therapy. Chemother Res Pract. 2012; 2012: 743193

70. Xu J, Acharya S, Sahin O, et al. 14-3-3zeta turns TGF-beta's function from tumor suppressor to metastasis promoter in breast cancer by contextual changes of Smad partners from p53 to Gli2. Cancer Cell. 2015; 27: 177-92.

71. Tang $Y$, Lv $P$, Sun $Z$, et al. 14-3-3 $\zeta$ up-regulates hypoxia-inducible factor-1 $a$ in hepatocellular carcinoma via activation of PI3K/Akt/NF-KB signal transduction pathway. Int J Clin Exp Pathol. 2015; 8: 15845-53.

72. Yang MR, Zhang Y, Wu XX, et al. Critical genes of hepatocellular carcinoma revealed by network and module analysis of RNA-seq data. Eur Rev Med Pharmacol Sci. 2016; 20: 4248-56.

73. Liu M, Liu X, Ren P, et al. A cancer-related protein 14-3-3zeta is a potential tumor-associated antigen in immunodiagnosis of hepatocellular carcinoma. Tumour Biol. 2014; 35: 4247-56.

74. Qin J, Wang S, Wang $\mathrm{P}$, et al. Autoantibody against $14-3-3$ zeta: a serological marker in detection of gastric cancer. J Cancer Res Clin Oncol. 2019; 145: 1253-62.

75. Zhang Y, Yang Y, Chen L, et al. Expression analysis of genes and pathways associated with liver metastases of the uveal melanoma. BMC Med Genet. 2014; 15: 29.

76. Huang Y-D, Shan W, Zeng L, et al. Screening of Differentially Expressed Genes Related to Bladder Cancer and Functional Analysis with DNA Microarray. Asian Pac J Cancer Prev. 2013; 14: 4553-7.

77. Tang Z, Li C, Kang B, et al. GEPIA: a web server for cancer and normal gene expression profiling and interactive analyses. Nucleic Acids Res. 2017; 45: W98-W102

78. Frasor J, Chang EC, Komm B, et al. Gene expression preferentially regulated by tamoxifen in breast cancer cells and correlations with clinical outcome. Cancer Res. 2006; 66: 7334-40.

79. Li Y, Zou L, Li Q, et al. Amplification of LAPTM4B and YWHAZ contributes to chemotherapy resistance and recurrence of breast cancer. Nat Med. 2010; 16: 214-8.

80. Bergamaschi A, Madak-Erdogan Z, Kim YJ, et al. The forkhead transcription factor FOXM1 promotes endocrine resistance and invasiveness in estrogen receptor-positive breast cancer by expansion of stem-like cancer cells. Breast Cancer Res. 2014; 16: 436. 
81. Matta A, Desouza LV, Shukla NK, et al. Prognostic significance of head-and-neck cancer biomarkers previously discovered and identified using iTRAQ-labeling and multidimensional liquid chromatography-tandem mass spectrometry. J Proteome Res. 2008; 7: 2078-87.

82. Yang X, Cao W, Zhou J, et al. 14-3-3zeta positive expression is associated with a poor prognosis in patients with glioblastoma. Neurosurgery. 2011; 68: 932-8; discussion 8.

83. Chatterjee D, Goldman M, Braastad CD, et al. Reduction of 9-nitrocamptothecin-triggered apoptosis in DU-145 human prostate cancer cells by ectopic expression of 14-3-3zeta. Int J Oncol. 2004; 25: 503-9.

84. Bai L, Zhou H, Xu R, et al. A Potent and Selective Small-Molecule Degrader of STAT3 Achieves Complete Tumor Regression In Vivo. Cancer Cell. 2019; 36: 498-511.e17.

85. Wei GY, Hu M, Zhao L, et al. MiR-451a suppresses cell proliferation, metastasis and EMT via targeting YWHAZ in hepatocellular carcinoma. Eur Rev Med Pharmacol Sci. 2019; 23: 5158-67. 